\title{
O estado da arte de pesquisas sobre a educação de surdos no Brasil de 2007 a 2011
}

\section{State of the art of research pieces on the education of deaf people in Brazil from 2007 to 2011}

\author{
Karina Soledad Pagnez ${ }^{1}$ \\ Cássia Geciauskas Sofiato ${ }^{1}$
}

\begin{abstract}
RESUMO
Este artigo apresenta os resultados de um estudo quantitativo-descritivo das pesquisas realizadas no Brasil no período de 2007 a 2011, com a busca realizada nos resumos de teses e dissertações disponíveis no Banco de Teses da Capes (www.capes.gov.br), tendo como palavras-chave: educação de surdos e Libras. A fim de analisar o corpus encontrado, foram definidas as seguintes categorias: (i) modalidade de produções, (ii) produções por ano, (iii) produções acadêmicas por universidades, (iv) áreas de concentração por ano, (v) temáticas escolhidas e quantidades por ano, (vi) tipos de pesquisa e quantidade por ano, (vii) referenciais teóricos relacionados à surdez e quantidades de citações por ano, (viii) órgãos de fomento à pesquisa. Destaca-se que, no período de cinco anos, foram defendidos 349 trabalhos, dentre os quais 281 dissertações de mestrado, 16 teses de doutorado e nove mestrados profissionais. A Universidade Federal de Santa Catarina foi o local em que foram realizadas 32 pesquisas no período, onde a área da Educação concentrou o maior número de trabalhos, totalizando 129, e a escolarização de surdos apresenta-se como temática recorrente com 30 trabalhos defendidos. $\mathrm{O}$ autor mais citado nos trabalhos é Vygotsky. De modo geral, os resumos apresentam limitações para a análise, o que impõe um desafio quanto à qualidade dos resumos das pesquisas que constam neste banco de dados.
\end{abstract}

Palavras-chave: educação de surdos; Libras; surdez; pesquisas.

DOI: $10.1590 / 0104-4060.33394$

1 Universidade de São Paulo, Faculdade de Educação. São Paulo, São Paulo, Brasil. Av. da Universidade, $\mathrm{n}^{\circ} 308$ - Butantã. CEP: 05508-040. 


\begin{abstract}
This study presents a quantitative-descriptive investigation of researches conducted in Brazil from 2007 to 2011. The search was conducted in the theses abstracts of masters' and doctorate programs in the Theses database at Capes (www.capes.gov.br), by using the keywords: Libras (Brazilian Sign Language) and Deaf people's education. The intention was to analyze the corpus found in the Theses database and with the following categories: (i) types of research, (ii) number of productions per year, (iii) academic production in each university, (iv) focus of fields per year, (v) themes chosen and amounts per year, (vi) kinds of research and amounts per year, (vii) theoretical reference on deafness and number of quotations per year, (viii) agencies for research funding. We emphasize that during five years 349 studies were presented and there were 281 Master's Theses, 16 Doctoral Theses and 9 works of professional master's. Santa Catarina Federal University was the institution where 32 researches were developed at the time, where the educational field had the biggest number of dissertations, representing 129 of the total amount, and deafs' education was presented as a recurring theme with 30 dissertations. Vygotsky was the author who was quoted in most of the studies. In general, the abstracts present analysis limitations and they pose a challenge to their quality.
\end{abstract}

Keywords: deaf people's education; Libras; deafness; research pieces.

\title{
Introdução
}

Para que se tenha um panorama acerca do estado da arte das pesquisas sobre a educação de surdos no Brasil no período delimitado para o estudo em questão (2007 a 2011), se faz necessário apresentar um breve contexto histórico que ofereça subsídios para refletirmos a respeito das primeiras produções relativas à educação de surdos no Brasil, desde os seus primeiros formatos, até as que serão produzidas no contexto das universidades atualmente.

Historicamente, a educação de surdos inicia-se no Brasil com a fundação oficial do Imperial Instituto dos Surdos-Mudos em 1857, atual Instituto Nacional de Educação de Surdos (INES). Neste instituto, em virtude de ter sido a primeira instituição para o ensino de surdos no Brasil, foram lançadas as bases para a educação desses alunos e, com o passar dos anos, tornou-se uma referência nacional.

Nos estudos realizados por Rocha (2007), constata-se que, durante a gestão de muitos diretores no Imperial Instituto de Surdos-Mudos, a prática 
de registro dos processos educacionais no formato de relatórios era frequente. De certa forma, além de informarem quanto à situação geral do Instituto e seu funcionamento, por meio de narrativas, esses documentos apresentavam indícios de como o processo relativo à educação de surdos era desenvolvido à época.

Essa prática teve início com E. Huet, primeiro diretor do Instituto, e foi continuada pelos seus sucessores. Exemplo disso é o documento intitulado $\mathrm{Me}$ mória de número $X X V$, da publicação Notícia Histórica dos Estabelecimentos Dependentes do Ministério da Justiça e Negócios Interiores, datado de 1898, destacado por Rocha (2007). Tal publicação e outras se constituem como fontes primárias para a pesquisa a respeito do Instituto nas quatro primeiras décadas de funcionamento.

Mazzotta (2001) destaca que a criação do Imperial Instituto dos Surdos-Mudos por parte do império, embora fosse uma medida precária em termos nacionais, pois inicialmente atendia um número pequeno de surdos comparado à população de surdos existentes, abriu a possibilidade para a discussão da educação das pessoas com deficiência, no $1^{\circ}$ Congresso de Instrucção Pública, em 1883, convocado pelo Imperador em dezembro de 1882.

Objetivamente, este congresso seria o primeiro evento que sistematizaria produções a respeito da educação de surdos. Mas esse congresso, segundo Schelbauer (2013), não chegou a se realizar em função da queda do gabinete Conservador e da substituição do Ministro do Império, Leão Veloso. Entretanto, os pareceres elaborados constituem documentos sobre as primeiras produções científicas da área. Os pareceres foram divididos em estudos: 29 relativos à instrução primária, secundária e profissional e 17 sobre a instrução superior.

Os autores dos pareceres, para a sua submissão, tinham que estar atentos às seguintes normas previstas no Regulamento do congresso: “[...] uma exposição dos factos atestados pelas estatísticas e relatórios concernentes a instrucção pública e terminar por uma proposta que sirva de base as discussões e deliberações do congresso" (CARVALHO, 1884, apud SCHELBAUER, 2013). No que se refere à produção sobre a área, a temática Educação dos surdos-mudos foi submetida na sessão intitulada Primeira Sessão: Instrução primária, secundária e profissional, pelos Drs. Tobias Leite e Menezes Vieira.

É interessante observar que a presença de um trabalho desta natureza em um congresso de grande porte evidencia a preocupação com a educação das pessoas com deficiência e, neste caso, especificamente, a educação dos surdos. Neste sentido, em que pese o congresso não ter ocorrido, os pareceres emitidos à época destacavam o estado da arte dos estudos em educação, Educação Especial e, além disso, evidenciavam o pensamento educacional brasileiro.

Rocha (2007) refere que, em 1926, o Dr. Arnaldo de Oliveira Bacellar, estudante da Faculdade de Medicina de São Paulo, publicou a sua tese de dou- 
toramento em medicina intitulada A Surdo-Mudez no Brasil. Segundo a mesma autora, a tese trazia temas referentes à surdez, tais como: "breve histórico sobre a questão da surdez e do surdo, etiologia, anatomopatologia, prevenção, legislação e, por fim, comentários sobre instituições brasileiras que atendem surdos” (p. 60). O autor do trabalho realizou uma pesquisa de campo no Instituto Nacional de Surdos-Mudos (antigo Imperial Instituto dos Surdos-Mudos) para levar a cabo tal projeto. Esta pesquisa é um exemplo das pesquisas desenvolvidas por décadas, cuja abordagem médica era central nas produções.

Mazzotta (2001) evidencia o surgimento de outras instituições destinadas à educação de surdos no Brasil a partir de 1929, entre elas: o Instituto Santa Terezinha em São Paulo (1929); a Escola Municipal de Educação Infantil e de $1^{\circ}$ Grau para deficientes auditivos Helen Keller (1951); o Instituto Educacional São Paulo, criado em 1954, que, em 1969, foi doado à Fundação São Paulo, entidade mantenedora da Pontifícia Universidade Católica de São Paulo, que com o passar do tempo tornou-se também um centro de referência para a pesquisa e o ensino de surdos.

Com o surgimento dessas e outras instituições no Brasil, as demandas relativas à produção de conhecimento sobre a educação de surdos consolidam-se, tornando-a objeto de pesquisas na área da saúde. Dentre as demandas mais prementes, podemos destacar a necessidade de material didático-pedagógico que subsidiasse as aulas (muitos textos foram traduzidos do francês para o português), as metodologias específicas para o ensino da fala e da linguagem e de disciplinas que compunham o currículo, a formação de professores para o trabalho com esse alunado, dentre outras temáticas.

Em virtude do trabalho empreendido, no seio dessas instituições surgem as primeiras publicações em forma de livros que tratam de aspectos análogos à educação de surdos e suas implicações (A Arte dos Surdos, em 1942; Vamos Falar: cartilha para o uso das crianças surdas brasileiras, em 1946; Quero Falar: cartilha para uso das crianças surdas - o Ensino da Articulação, em 1957). Nota-se que, também, a imprensa brasileira, representada pelos jornais Diário Carioca, Diário de Notícias, Jornal do Brasil, Correio da manhã e Folha da Noite, relatava as iniciativas/acontecimentos que envolviam os surdos e as instituições que se dedicavam a essa causa.

Somado a tudo isso, vemos surgir de forma mais incisiva o interesse acadêmico por questões referentes à educação de surdos e seu desenvolvimento linguístico a partir da segunda metade da década de 1980. Este fato se deu também em virtude do trabalho desenvolvido por William Stokoe, linguista americano, em 1960, a respeito da língua americana de sinais (ASL), pois as línguas de sinais passaram a ter o reconhecimento linguístico e isso mudou ra- 
dicalmente as concepções que se tinha até então sobre tal língua e sua influência no desenvolvimento da pessoa surda.

Com base em Felipe (2008), destacamos que pesquisas linguísticas sobre a língua brasileira de sinais (Libras) passam a fazer parte do cenário acadêmico e de discussões de grupos de pesquisas afins, além dos temas relacionados à educação de surdos. Tanto que o primeiro registro no banco de teses da Coordenação de Aperfeiçoamento de Pessoal de Nível Superior (CAPES) sobre o tema surdez data de 1987, configura-se como uma dissertação de mestrado e traz como tema: A criança surda: educação para a marginalização.

Na década de 1990, há um crescimento em relação ao número de trabalhos acadêmicos publicados, seja em forma de dissertação de mestrado, teses de doutorado, artigos científicos ou livros, e muitos abordavam aspectos referentes à Libras.

Com a promulgação da Lei ${ }^{\circ} 10.432$, de 2002, a língua brasileira de sinais passa a ser reconhecida como língua oriunda de comunidades de pessoas surdas do Brasil. E por meio do Decreto $\mathrm{n}^{\circ}$ 5.626, de 2005, tal lei é regulamentada e ainda há, em nove capítulos, a disposição da língua em questão, enfatizando-se os aspectos da educação e da saúde.

Esses fatos, além de serem historicamente importantes e marcantes para a área da surdez e educação de surdos, pois asseguram os direitos da comunidade surda em vários âmbitos e servem de referência para os movimentos sociais, engendram novos estudos e pesquisas a partir do momento em que surgem.

Com este panorama de fundo, a pesquisa objetiva identificar e descrever as produções acadêmicas relativas à educação de pessoas surdas de 2007 a 2011, a partir de um banco de dados específico (banco de teses da CAPES). A escolha deste recorte temporal deu-se em função de que a produção na área nos últimos cinco anos apresentou um aumento progressivo e constante. Por meio dessa empreitada, intentamos fornecer subsídios para o avanço das pesquisas nessa área temática.

\section{Método}

Esta pesquisa se configura como quantitativa e descritiva. De acordo com Gil (1996), "as pesquisas descritivas têm como objetivo primordial a descrição das características de determinada população ou fenômeno, ou, então, o estabelecimento de relações entre variáveis" (p. 46). 
Para a realização da coleta de dados, foi consultado o Banco de Teses da Coordenação de Aperfeiçoamento de Pessoal de Nível Superior (CAPES), que disponibiliza os resumos pela internet e no qual constam teses e dissertações aprovadas nos cursos brasileiros de pós-graduação no período de 1987 a 2011. Neste trabalho, definiu-se um período de cinco anos para a coleta de dados, o período foi demarcado no extrato final pelo último ano em que teríamos disponíveis as informações no Banco de Teses, ou seja, 2011, e retrocedendo-se cinco anos, temos como corte inicial o ano de 2007. Dessa forma, os dados analisados estão compreendidos entre os anos de 2007 e 2011.

O acesso ocorreu nos meses de fevereiro e março de 2013. Para a padronização e organização da busca, foram utilizadas as seguintes palavras/ expressões-chave: educação de surdos e Libras.

Os trabalhos foram selecionados inicialmente pelo título e, a partir da sua inclusão por este critério, foi realizada a leitura do resumo, de onde foram retiradas informações pertinentes à pesquisa. Cabe ressaltar que uma significativa parte dos resumos apresentava limitações de ordem estrutural, formal e teórica.

Para a organização e investigação dos dados coletados, foram definidas oito categorias de análise: (i) modalidade de produções, (ii) produções por ano, (iii) produções acadêmicas por universidades, (iv) áreas de concentração por ano, (v) temáticas escolhidas e quantidades por ano, (vi) tipos de pesquisa e quantidade por ano, (vii) referenciais teóricos relacionados à surdez e quantidades de citações por ano, (viii) órgãos de fomento à pesquisa.

A sistematização dos dados ocorreu a partir de tabelas e gráficos de barra e de setor por considerar-se que os dados neles apresentados consolidam uma análise quantitativa e descritiva.

\section{Apresentação dos resultados}

Antes de apresentarmos os dados referentes ao recorte temporal delimitado para esta pesquisa, destacamos alguns aspectos gerais referentes aos trabalhos que compõem o Banco de Teses da CAPES relacionados às palavras-chave utilizadas na busca.

$\mathrm{O}$ ano em que se inicia a indexação das produções no Banco de Teses da CAPES é 1987. Entretanto, com base no levantamento realizado, verificamos que a produção, com base nas palavras-chave educação de surdos e Libras, teve início somente no ano de 1994. Assim, no período de 1994 a 2011, obtivemos como resultado do levantamento 679 pesquisas. 
No período definido para esta pesquisa - 2007 a 2011 - temos 349 produções, que estão divididas em mestrado, mestrado profissional e doutorado.

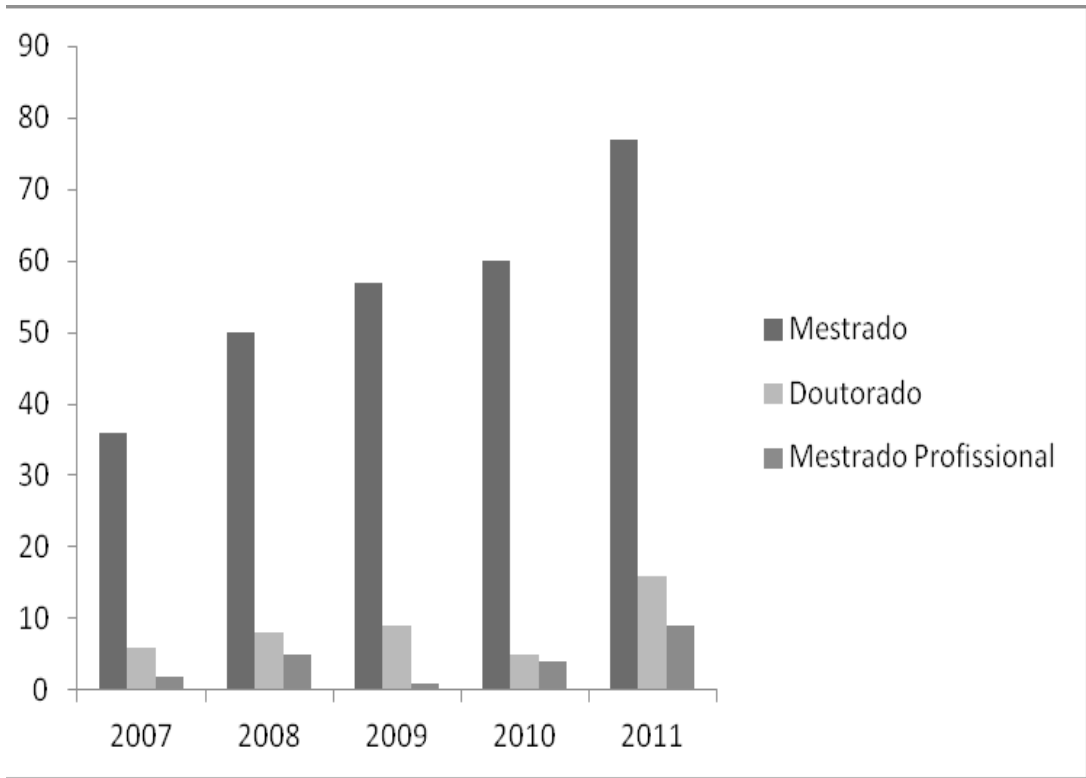

GRÁFICO 1 - MODALIDADES DE PRODUÇÕES DE 2007 A 2011

FONTE: Elaborado pelas autoras a partir do Banco de teses da CAPES

O gráfico 1 expressa o crescimento na produção de pesquisas na área de educação de surdos no período investigado. A quantidade de pesquisas de mestrado teve um crescimento da ordem de $100 \%$ nos dois extremos do recorte. O número de pesquisas de doutorado cresceu na ordem de $200 \%$ nos dois extremos, mas apresenta oscilações e não é um crescimento contínuo como ocorre nas pesquisas de mestrado. Devemos levar em consideração que o número de trabalhos de doutorado defendidos é significativamente inferior ao número de mestrados defendidos no mesmo período. O número de mestrados profissionais é inferior a dez pesquisas em todo o período, mesmo que tenha demonstrado crescimento no último ano. 
TABELA 1 - PRODUÇÃO POR ANO

\begin{tabular}{|c|c|c|c|c|}
\hline Ano & Mestrado & Doutorado & $\begin{array}{c}\text { Mestrado } \\
\text { Profissional }\end{array}$ & Totais por ano \\
\hline 2007 & 36 & 6 & 2 & 44 \\
\hline 2008 & 50 & 8 & 5 & 63 \\
\hline 2009 & 57 & 11 & 1 & 69 \\
\hline 2010 & 61 & 5 & 5 & 71 \\
\hline 2011 & 77 & 16 & 9 & 102 \\
\hline Total Geral & $\mathbf{2 8 1}$ & $\mathbf{4 6}$ & $\mathbf{2 2}$ & $\mathbf{3 4 9}$ \\
\hline
\end{tabular}

FONTE: Elaborada pelas autoras a partir do Banco de teses da CAPES

Foram defendidos, no período de cinco anos, 281 trabalhos de mestrado, 46 de doutorado e 22 de mestrado profissionalizante, perfazendo um total de 349 produções.

No período de 2007 a 2011, as produções relacionadas às três modalidades em questão foram realizadas em 83 universidades (Anexo 1). A seguir, são apresentadas as instituições que apresentaram número maior de pesquisas realizadas:

TABELA 2 - PRODUÇÕES ACADÊMICAS POR UNIVERSIDADES

\begin{tabular}{|l|c|c|c|c|c|c|}
\hline \multicolumn{1}{|c|}{ Universidades } & $\mathbf{2 0 0 7}$ & $\mathbf{2 0 0 8}$ & $\mathbf{2 0 0 9}$ & $\mathbf{2 0 1 0}$ & $\mathbf{2 0 1 1}$ & $\begin{array}{c}\text { Total de } \\
\text { produções }\end{array}$ \\
\hline $\begin{array}{l}\text { Pontifícia Universidade } \\
\text { Católica de São Paulo }\end{array}$ & 2 & 5 & 4 & 1 & 4 & $\mathbf{1 6}$ \\
\hline Universidade de Brasília & 2 & 4 & & 4 & 5 & $\mathbf{1 5}$ \\
\hline Universidade de São Paulo & 2 & 3 & 5 & 3 & 2 & $\mathbf{1 5}$ \\
\hline $\begin{array}{l}\text { Universidade Federal de Santa } \\
\text { Catarina }\end{array}$ & 4 & 6 & 4 & 11 & 7 & $\mathbf{3 2}$ \\
\hline
\end{tabular}

FONTE: Elaborada pelas autoras a partir do Banco de teses da CAPES

A Universidade Federal de Santa Catarina concentra o maior número de trabalhos defendidos no período investigado, em um total de 32 pesquisas. As demais universidades apresentam números acima de dez trabalhos. As outras 79 universidades apresentam menos de dez produções no período analisado, e é possível constatar que 27 dessas universidades têm apenas um trabalho defendido no período de cinco anos.

Dentre as quatro universidades destacadas, temos duas da região Sudeste, uma da região Sul e uma da região Centro-Oeste. 
No que tange as áreas de concentração das produções, respeitou- se a denominação presente nos trabalhos e, dessa forma, não foram realizados agrupamentos. Em relação a este aspecto, foram encontradas 58 áreas de concentração (anexo 2). Na tabela a seguir, são apresentadas as áreas que têm produção em no mínimo quatro dos anos do recorte definido e perfazendo um total acima de oito trabalhos no período:

TABELA 3 - ÁREAS DE CONCENTRAÇÃO POR ANO

\begin{tabular}{|c|c|c|c|c|c|c|}
\hline Área de concentração & $\mathbf{2 0 0 7}$ & $\mathbf{2 0 0 8}$ & $\mathbf{2 0 0 9}$ & $\mathbf{2 0 1 0}$ & $\mathbf{2 0 1 1}$ & Totais \\
\hline Ciência da Computação & 2 & 1 & 2 & 1 & 2 & $\mathbf{8}$ \\
\hline Educação & 9 & 21 & 33 & 24 & 42 & $\mathbf{1 2 9}$ \\
\hline Educação Especial & 5 & 3 & 4 & 2 & 5 & $\mathbf{1 9}$ \\
\hline Ensino de Ciências e Matemática & 2 & 3 & 4 & 3 & & $\mathbf{1 2}$ \\
\hline Fonoaudiologia & 3 & 4 & & 2 & 1 & $\mathbf{1 0}$ \\
\hline Letras & 2 & 2 & 1 & 4 & 2 & $\mathbf{1 1}$ \\
\hline Linguística & 2 & 8 & 7 & 9 & & $\mathbf{2 6}$ \\
\hline Linguística Aplicada & 1 & 6 & 4 & 2 & 3 & $\mathbf{1 6}$ \\
\hline Linguística, Letras e Artes & 2 & 1 & 4 & 1 & 5 & $\mathbf{1 3}$ \\
\hline
\end{tabular}

FONTE: Elaborada pelas autoras a partir do Banco de teses da CAPES

Dentre os 349 trabalhos defendidos no período investigado, 129 foram realizados na área de Educação, a área da Linguística somada às três modalidades perfaz 55 trabalhos; há ainda números expressivos nas áreas de Letras, Fonoaudiologia e Educação Especial. Para as pesquisadoras, duas áreas que se destacam são Ciência da Computação e Ensino de Ciências e Matemática, as quais a princípio não eram consideradas áreas de pesquisa voltadas para esta temática. Nas demais 49 áreas, foram defendidas apenas duas ou três pesquisas.

Considerou-se também apresentar uma análise das temáticas presentes nos trabalhos. Ademais, é fundamental apontar sua recorrência no corpus da pesquisa.

A definição das temáticas resultou da leitura dos objetivos descritos pelos autores nos resumos das pesquisas. Ao longo do período observado, destaca-se a consolidação de 101 temáticas (anexo 3). Conforme o proposto quanto à área temática, foi respeitado o que o autor definiu como temática, o que num primeiro olhar configura possibilidades de agrupamento, porém manteve-se como constava nos trabalhos. Com a leitura e releitura dos resumos, não consideramos pertinente para a consolidação do estado da arte definir temáticas a priori, mas, pelo contrário, trazer o que dizem as pesquisas com a maior fidedignidade. 
TABELA 4 - TEMÁTICAS ESCOLHIDAS E QUANTIDADE POR ANO

\begin{tabular}{|c|c|c|c|c|c|c|}
\hline Temática & 2007 & 2008 & 2009 & 2010 & 2011 & Totais \\
\hline $\begin{array}{l}\text { Aquisição da língua } \\
\text { portuguesa escrita }\end{array}$ & - & 4 & 2 & - & - & 6 \\
\hline $\begin{array}{l}\text { Aquisição do português } \\
\text { por surdos }\end{array}$ & 3 & 4 & 3 & 1 & 6 & 17 \\
\hline Atuação do tradutor intérprete & 1 & & 1 & 3 & 1 & 6 \\
\hline Bilinguismo & - & 4 & - & 2 & 4 & 10 \\
\hline $\begin{array}{c}\text { Concepções ou } \\
\text { representações sobre pessoas } \\
\text { surdas }\end{array}$ & 1 & - & 2 & 1 & 9 & 13 \\
\hline Educação Superior/surdos & 2 & 1 & 1 & - & 4 & 8 \\
\hline Ensino de LIBRAS & 5 & - & - & 1 & 7 & 13 \\
\hline Ensino de matemática & 2 & 1 & 3 & 3 & - & 9 \\
\hline $\begin{array}{c}\text { Ensino do português escrito a } \\
\text { alunos surdos }\end{array}$ & 4 & 2 & 3 & 1 & 2 & 12 \\
\hline $\begin{array}{c}\text { Escolarização de pessoas } \\
\text { surdas }\end{array}$ & - & 2 & 4 & 1 & 23 & 30 \\
\hline $\begin{array}{c}\text { Escrita do português por } \\
\text { surdos }\end{array}$ & - & - & 1 & 5 & - & 6 \\
\hline Estudos Culturais & - & 1 & - & 4 & 2 & 7 \\
\hline Estudos Surdos & 2 & - & - & 2 & 3 & 7 \\
\hline Inclusão escolar & 1 & 5 & 2 & - & - & 8 \\
\hline $\begin{array}{l}\text { Informática e ensino de } \\
\text { surdos } \\
\end{array}$ & 1 & 2 & 2 & 2 & - & 7 \\
\hline Linguística da LIBRAS & - & 5 & 1 & 2 & 4 & 12 \\
\hline $\begin{array}{c}\text { Políticas educacionais e } \\
\text { surdez }\end{array}$ & 2 & 3 & 2 & - & - & 7 \\
\hline Psicologia e surdez & 4 & 2 & - & - & - & 6 \\
\hline
\end{tabular}

FONTE: Elaborada pelas autoras a partir do Banco de teses da CAPES

Na Tabela 4, constam as temáticas que apresentaram dentro do corpus um número igual ou maior a seis trabalhos. Das 101 temáticas, 18 delas respeitam esse critério; a temática Escolarização de Surdos está presente em 30 pesquisas; portanto, temos $10 \%$ das pesquisas defendidas que dizem respeito a esta temática. A temática Aquisição do português por surdos tem um total de 17 pesquisas.

Outra categoria de análise refere-se ao tipo de pesquisa definida no resumo do trabalho. Gil (1996) destaca que uma pesquisa, ao ser desenvolvida, deve levar em consideração "os conhecimentos disponíveis e a utilização cuidadosa 
de métodos, técnicas e outros procedimentos científicos" (p. 19). Assim sendo, temos a seguinte configuração:

TABELA 5 - TIPOS DE PESQUISA POR ANO E QUANTIDADE

\begin{tabular}{|c|c|c|c|c|c|c|}
\hline Tipologia & $\mathbf{2 0 0 7}$ & $\mathbf{2 0 0 8}$ & $\mathbf{2 0 0 9}$ & $\mathbf{2 0 1 0}$ & $\mathbf{2 0 1 1}$ & Totais \\
\hline Estudo de caso & & 4 & 4 & 4 & 2 & $\mathbf{1 4}$ \\
\hline Etnográfica & 2 & 5 & 1 & 5 & 4 & $\mathbf{1 7}$ \\
\hline Experimental & 1 & & & & 6 & $\mathbf{7}$ \\
\hline Pesquisa-Ação & 1 & 1 & 2 & & 2 & $\mathbf{6}$ \\
\hline Qualitativa & 3 & 7 & 8 & 6 & 9 & $\mathbf{3 3}$ \\
\hline Sem descrição & 31 & 34 & 47 & 54 & 69 & $\mathbf{2 3 5}$ \\
\hline
\end{tabular}

FONTE: Elaborada pelas autoras a partir do Banco de teses da CAPES

Diante de um corpus de 349 resumos de pesquisa, foram definidas 25 tipologias de pesquisa diferentes com destaque para o fato de que, em 235 pesquisas, não é definido ou especificado o tipo de pesquisa realizado.

A categoria referenciais teóricos foi definida como central para este estado da arte, e busca-se identificar os autores da área de surdez que têm subsidiado as pesquisas. Portanto, não serão elencados autores de outras áreas, tais como filosofia, sociologia, política, metodologia de pesquisa e outras. Tampouco serão elencados os autores citados sem serem assumidos como referenciais para a análise dos dados coletados.

Quanto aos referenciais teóricos da área da surdez, temos 33 itens (anexo 5). A seguir, são apresentados os que foram utilizados em, no mínimo, quatro dos anos definidos como recorte da pesquisa:

TABELA 6 - REFERENCIAIS TEÓRICOS RELACIONADOS À SURDEZ E QUANTIDADE DE CITAÇÕES POR ANO

\begin{tabular}{|c|c|c|c|c|c|c|}
\hline Referencial & $\mathbf{2 0 0 7}$ & $\mathbf{2 0 0 8}$ & $\mathbf{2 0 0 9}$ & $\mathbf{2 0 1 0}$ & $\mathbf{2 0 1 1}$ & Totais \\
\hline Perlin & & 1 & 2 & 1 & 2 & $\mathbf{6}$ \\
\hline Quadros & 1 & 2 & 1 & 2 & 5 & $\mathbf{1 1}$ \\
\hline Skliar & & 3 & 3 & 5 & 4 & $\mathbf{1 5}$ \\
\hline Vygotsky & 2 & 8 & 6 & 7 & 10 & $\mathbf{3 3}$ \\
\hline $\begin{array}{c}\text { Sem referências da } \\
\text { área de surdez }\end{array}$ & 36 & 44 & 54 & 56 & 54 & $\mathbf{2 4 4}$ \\
\hline
\end{tabular}

FONTE: Elaborada pelas autoras a partir do Banco de teses da CAPES 
Do total de 349 resumos de pesquisa, em 244 resumos não constam referências da área de surdez e, na grande maioria dos resumos, não constam referências. O trabalho de Vygotsky foi o referencial teórico mais citado e, em seguida, temos a menção a Skliar.

Finalmente, a última categoria analisada nesta pesquisa diz respeito ao financiamento declarado pelos pesquisadores nos resumos de suas pesquisas. Optou-se por uma apresentação em gráfico. Esta escolha se justifica pela necessidade de visualização das especificidades de cada ano.

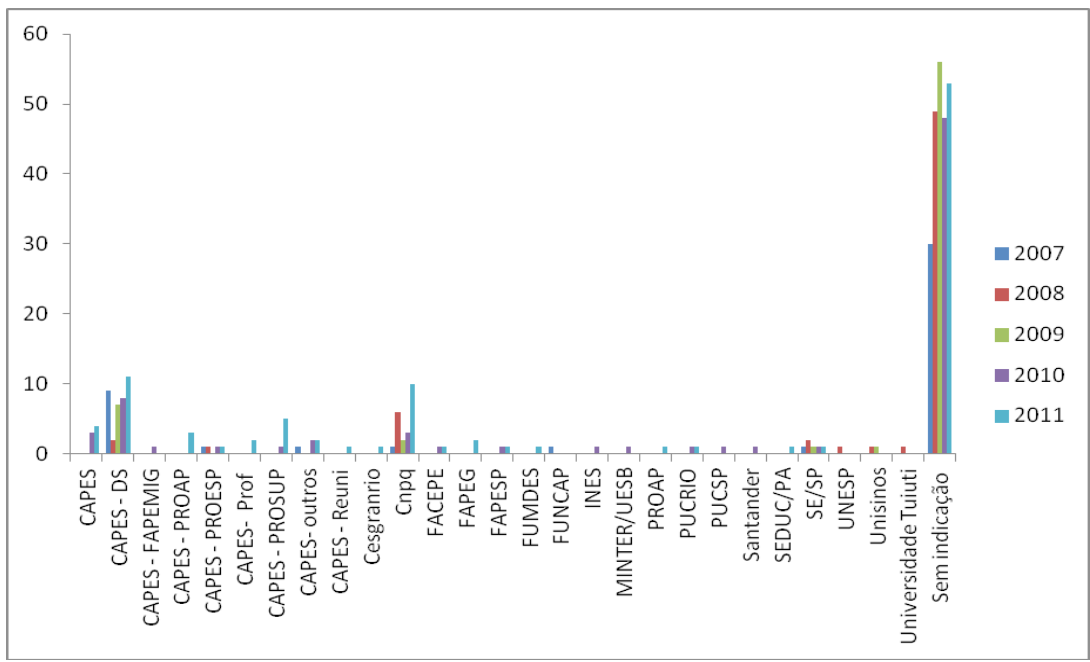

GRÁFICO 2 - ÓRGÃOS DE FOMENTO À PESQUISA

FONTE: Elaborado pelas autoras a partir do Banco de teses da CAPES

Constata-se, pelo gráfico, que um número significativo de pesquisas não recebe financiamento. $\mathrm{O}$ ano de 2009 foi o que expressou maior número. Um crescimento no número de pesquisas financiadas delineia-se em 2011. A agência que tem assumido esta responsabilidade de forma efetiva é a CAPES. Destaca-se também que algumas universidades privadas optaram por assumir o financiamento de pesquisas.

Atualmente, por volta de $50 \%$ das pesquisas recebe financiamento. Ademais, é expressivo o aumento do número de agências e de linhas de financiamento dentro de uma mesma agência. No ano de 2011, a CAPES apresentou oito linhas de financiamento. 


\section{Discussão dos resultados}

O estudo revela que a temática da educação de surdos tem se consolidado como área de interesse para investigação em diferentes universidades, ocorrendo em diferentes áreas temáticas. Em relação aos aspectos formais referentes à pesquisa, ao realizarmos nossas buscas no banco de Teses da CAPES, foram encontradas limitações nos resumos de pesquisas.

Cabe ressaltar o que Maciel e Vieira (2007) recuperam da orientação de Severino (2002), em relação à composição de um resumo de trabalho acadêmico:

De que natureza é o trabalho analisado (pesquisa empírica, pesquisa teórica, levantamento documental, pesquisa histórica, etc.)? Qual o objeto pesquisado/estudado? O que se pretende demonstrar ou constatar? Em que referências teóricas se apoiou o desenvolvimento do raciocínio? Mediante quais procedimentos técnico-operacionais se procedeu? Quais os resultados conseguidos em termos de atingimento dos objetivos propostos? (p. 173)

Tendo como base a citação anterior e as análises realizadas, observam-se diferentes limitações: inexistência de um dos resumos; resumos com apenas uma linha, o que não nos permitiu a análise; a presença de resumos que em nada se relacionavam com o título proposto na pesquisa, fato que também impossibilitou as suas análises. Além disso, encontramos problemas de natureza formal em muitos resumos analisados. Entre eles, podemos destacar a variedade de tamanhos dos resumos encontrados no Banco de Teses e os erros relativos à língua portuguesa encontrados durante o percurso da pesquisa.

Ao realizar o levantamento no Banco de Teses, foram lançadas as palavras-chave e os resultados da busca apresentaram uma série de trabalhos que não tinham relação com os termos utilizados. Como exemplo, temos os seguintes títulos: A percepção da qualidade da informação na área de silvicultura da Klabin; Sistema de gestão ambiental: um estudo dos terminais do porto de Santos.

Verificou-se ainda que muitos resumos não apresentavam o tipo da pesquisa, seja a partir de critérios como natureza, objetivos ou procedimentos. $\mathrm{Na}$ maioria dos trabalhos, os pesquisadores fazem uma descrição dos procedimentos e nomeiam as técnicas utilizadas. Em sua maioria, as técnicas de coleta de dados foram entrevistas ou observações. Isso denota uma confusão entre mencionar 
o tipo de pesquisa a ser desenvolvido e a classificação das pesquisas quanto à abordagem do problema e a descrição do método.

Outro ponto preocupante nos resumos diz respeito ao referencial teórico. Em 244 trabalhos, não foi explicitada a matriz teórica e nem foram citados autores da área da surdez. Há casos em que os autores colocam: "a base teórica do trabalho nos permite verificar que [...]", sem a indicação dessa base teórica. Cabe retomar o que escreve Severino (2002) quanto às referências teóricas. Para o referido autor, elas são os subsídios para o desenvolvimento do raciocínio da pesquisa, portanto, são imprescindíveis. Isso nos remete a uma reflexão quanto ao desenvolvimento do raciocínio das pesquisas, apontando para uma possível fragilidade.

Quanto às técnicas utilizadas e instrumentos de coleta de dados, foram citados: entrevistas, observações, produção escrita, gravações, documentos, registros escritos, dentre outros. Os dados coletados não justificavam a inclusão desta categoria.

Todos esses problemas destacados em relação à composição dos resumos interferiram no andamento desta investigação, exigindo um cuidado bastante grande em relação à busca das informações e categorização dos dados.

Maciel e Vieira (2007), ao analisarem os resumos do Banco de Teses da CAPES, no período de 1987 a 2005, encontraram problemas semelhantes aos relatados anteriormente. E esses autores fazem um questionamento que se toma de empréstimo: "é possível se cumprir, com efetividade, o papel designado a um banco de dados diante desses e tantos outros problemas?" (p. 361).

Em relação às análises das categorias propostas, considera-se que, em relação ao volume de produção relativo às pesquisas de mestrado, doutorado e mestrado profissional, houve um crescimento significativo em cada uma delas, destacando-se que o número de pesquisas de doutorado e mestrado profissionalizante foi expressivamente menor que o número de pesquisas de mestrado. Considera-se que o volume de doutorados defendidos neste período não expressa a continuidade de estudos nesse nível de alunos formados no mestrado, que tiveram como eixo de pesquisa a educação de surdos e a Libras.

Quanto à universidade que se destaca pelo número de defesas, a Federal de Santa Catarina tem mantido um número expressivo de defesas por ano. Em geral, as pesquisas têm se concentrado nas regiões Sul e Sudeste e encontram-se distribuídas por diferentes áreas temáticas, com a prevalência de pesquisas na área da Educação, com destaque para áreas em que, a princípio, não se esperariam trabalhos sobre essa temática, por exemplo, as da engenharia elétrica e do design. Além disso, as temáticas como: ensino de matemática, ensino de física, ensino de química e ensino de ciências demonstram uma preocupação com o ensino de ciências para surdos. 
Dentre as pesquisas investigadas, constata-se uma prevalência quanto à Escolarização de Surdos. Em algumas pesquisas, essa temática é definida pelo autor; em outras, as discussões a tangenciam. No que se refere ao tipo de pesquisa, há uma predominância dos estudos que não descrevem tipo de pesquisa. Dentre os que realizam esta descrição, temos uma maior incidência de estudos de caso e das pesquisas etnográficas.

Quantos aos autores da surdez utilizados como referencial teórico das pesquisas, temos a citação de Vygotsky e Quadros nos cinco anos investigados e, em relação a Skliar, as citações ocorrem a partir do ano de 2008.

Uma questão central para o desenvolvimento de pesquisas refere-se ao financiamento. Nas pesquisas analisadas, destaca-se que, em 2007, uma parcela pequena contou com apoio de agências de fomento. Em 2008, ocorreu uma diminuição deste investimento e, em 2009, houve uma diminuição em relação ao ano anterior. Já em 2010, destaca-se um aumento em relação aos anos anteriores; em 2011, passa-se a ter financiamento em quase $50 \%$ das pesquisas. Estes dados são animadores para o aumento de pesquisas na área, o que consolida a produção.

\section{Conclusões}

A pesquisa em questão nos permitiu conhecer a evolução das produções referentes à educação de surdos nos últimos cinco anos. O histórico apresentado sobre as primeiras produções, que obedeciam a uma lógica da área médica, mesmo sendo desenvolvidas em instituições definidas como educacionais, permite que se constate a mudança na proposta e a consolidação de pesquisa fundamentalmente na e da área de educação; o abandono de uma abordagem médico-pedagógica, como define Mazzotta (2001), e a consolidação de pesquisas sócio-históricas ou socioantropológicas iniciadas no século XX.

Observa-se que os registros referentes ao processo educacional dos sujeitos surdos ao longo da história se deram por meio de relatórios, pareceres, livros e artigos em periódicos, até chegarmos às produções geradas nas instituições de ensino superior, em forma de dissertações de mestrado e teses de doutorado. Em que pese o fato de ainda contarmos com variados tipos de produções na área da surdez, as dissertações e as teses, a partir do momento em que começam a surgir no contexto das universidades, também promovem a divulgação do conhecimento a partir das pesquisas engendradas.

A partir da indexação das dissertações e teses no Banco de Teses da CAPES, apesar dos problemas técnicos constatados neste estudo, a circulação 
dessas produções passa a ser mais efetiva, partindo-se do princípio de que o acesso a essa base é irrestrito e amplo, o que consolida um desafio para as instituições em que são defendidas as dissertações e teses: a qualidade dos resumos nela inseridos. É fundamental que os resumos apresentem elementos centrais do trabalho científico que possibilitem que outros pesquisadores conheçam as produções e encontrem interlocutores para suas investigações.

A partir das análises realizadas no período proposto, observa-se que a produção relacionada às áreas de educação de surdos e Libras tem sido constante em universidades públicas e privadas e perpassa todas as regiões brasileiras. As agências de fomento de pesquisa têm uma parcela de participação neste processo. Entretanto, o financiamento é outro desafio para as pesquisas e sua consolidação.

A caracterização de cinco anos de produção aponta temas prevalentes, temas silenciados e, principalmente, subsidia, a partir do que já foi produzido, novas investigações na área.

\section{REFERÊNCIAS}

BRASIL, Ministério da Educação. Secretaria da Educação Especial. Lei no 10.436, de 24 de abril de 2002. Dispõe sobre a Língua Brasileira de Sinais- LIBRAS e dá outras providências. Diário Oficial da União, Brasília, DF, 25 abr. 2002. Disponível em: $<$ http:// www.planalto.gov.br/ccivil_03/leis/2002/110436.htm>. Acesso em: 11/04/2013.

BRASIL, Ministério da Educação. Secretaria da Educação Especial. Decreto n ${ }^{0} 5.626$, de 22 de dezembro de 2005. Regulamenta a Lei $\mathrm{n}^{\circ} 10.436$, de 24 de abril de 2002. Diário Oficial da União, Brasília, DF, 23 dez. 2005. Disponível em: <http://www.planalto.gov. br/ccivil_03/_ato2004-2006/2005/decreto/d5626.htm>. Acesso em: 10/04/2013.

CAPES. Coordenação de Aperfeiçoamento de Pessoal de Nível Superior. Serviços: Banco de teses. 2005. Disponível em: $<$ http://servicos.capes.gov.br/capesdw/>. Acesso em: fevereiro/março/2013.

CARVALHO, Carlos Leôncio de. Primeira exposição pedagogica do Rio de Janeiro: Relatorio. Rio de janeiro: Typographia Nacional, 1884.

FELIPE. T. De Flausino ao Grupo de Pesquisa da FENEIS - RJ. Anais do V Seminário Nacional do INES. Rio de Janeiro: INES, 2008.

GIL, A.C. Como elaborar projetos de pesquisa. São Paulo: Atlas, 1996.

MACIEL, L. S. B, VIEIRA, R. de A. Fonte investigadora em Educação: registros do banco de teses da CAPES. Disponível em: $<$ http://www.scielo.br/pdf/ep/v33n2/a12v33n2. pdf $>$. Acesso em: 11/04/2013. 
MAZZOTTA, M. J. S. Educação Especial no Brasil: história e políticas. São Paulo: Cortez, 2001.

ROCHA, S. O INES e a educação de surdos no Brasil: aspectos da trajetória do Instituto Nacional de Educação de Surdos em seu percurso de 150 anos. Rio de Janeiro: INES, 2007.

SCHELBAUER, A. R. Fonte para o estudo da história da educação brasileira: o congresso da instrução do Rio de Janeiro. Disponível em: <http://www.sbhe.org.br/novo/ congressos/cbhe1/anais/022_analete_regina.pdf>. Acesso em: 07/04/ 2013.

SEVERINO, A. J. Metodologia do trabalho cientifico. 22. ed. São Paulo: Cortez, 2002.

Texto recebido em 15 de setembro de 2013.

Texto aprovado em 10 de março de 2014. 


\section{ANEXO 1}

TABELA 2 - PRODUÇÕES ACADÊMICAS POR UNIVERSIDADES

\begin{tabular}{|c|c|c|c|c|c|}
\hline Universidades & 2007 & 2008 & 2009 & 2010 & 2011 \\
\hline Centro de Ensino Superior de Juiz de Fora & 1 & & & & \\
\hline $\begin{array}{l}\text { Centro Federal de Educação Tecnológica Celso } \\
\text { Suckow da Fonseca }\end{array}$ & & & 1 & 1 & \\
\hline Centro Universitário Franciscano & 1 & & & & \\
\hline Centro Universitário La Salle & & & 2 & & \\
\hline Centro Universitário Moura Lacerda & 1 & 2 & 2 & 3 & 1 \\
\hline Centro Universitário Plínio Leite & & 4 & & & \\
\hline Centro Universitário Salesiano de São Paulo & 2 & & & & \\
\hline Centro Universitário Univates & & & & 1 & \\
\hline Fundação Cesgranrio & & & & & 1 \\
\hline Fundação de Ensino Eurípedes Soares da Rocha & 1 & & & & \\
\hline $\begin{array}{l}\text { Fundação Universidade Federal do Mato Grosso } \\
\text { do Sul }\end{array}$ & & 1 & & 1 & \\
\hline $\begin{array}{l}\text { Instituto Federal de Educação Ciência e Tecnologia } \\
\text { do Rio de Janeiro }\end{array}$ & & & & & 1 \\
\hline Instituto Tecnológico da Aeronáutica & & 1 & & & \\
\hline Pontifícia Universidade Católica de Campinas & & & & 1 & \\
\hline Pontifícia Universidade Católica de Goiás & 1 & 1 & & & 1 \\
\hline Pontifícia Universidade Católica de Minas Gerais & & & 1 & & \\
\hline Pontifícia Universidade Católica de São Paulo & 2 & 5 & 4 & 1 & 4 \\
\hline Pontifícia Universidade Católica do Paraná & & & & 1 & \\
\hline Pontifícia Universidade Católica do Rio de Janeiro & & 1 & 2 & & 2 \\
\hline $\begin{array}{l}\text { Pontifícia Universidade Católica do Rio Grande } \\
\text { do Sul }\end{array}$ & 1 & & 1 & 1 & \\
\hline Universidade Federal do Rio Grande & & & & & 2 \\
\hline Universidade Católica de Brasília & & & 4 & 1 & 2 \\
\hline Universidade Católica de Pernambuco & 1 & 1 & 1 & 1 & \\
\hline Universidade Católica Dom Bosco & & 1 & & & \\
\hline Universidade de Brasília & 2 & 4 & & 4 & 5 \\
\hline
\end{tabular}

(continua) 
(continuação)

\begin{tabular}{|c|c|c|c|c|c|}
\hline Universidade de Caxias do Sul & & & & & 2 \\
\hline Universidade de Fortaleza & 1 & & & & \\
\hline Universidade de São Paulo & 2 & 3 & 5 & 3 & 2 \\
\hline Universidade de Taubaté & & 1 & & 1 & \\
\hline Universidade do Estado da Bahia & & & 1 & & \\
\hline Universidade do Vale do Rio dos Sinos & & 1 & 2 & 1 & 2 \\
\hline Universidade Estácio de Sá & 1 & & & & 2 \\
\hline Universidade Estadual de Campinas & & 3 & 3 & & 2 \\
\hline Universidade Estadual de Londrina & & & 1 & & 1 \\
\hline Universidade Estadual de Maringá & & 1 & 1 & 1 & 1 \\
\hline Universidade Estadual do Ceará & & 1 & 2 & 2 & 4 \\
\hline Universidade Estadual do Oeste do Paraná & & & & 1 & \\
\hline Universidade Estadual do Pará & & & & 2 & 2 \\
\hline Universidade Estadual do Paraná & & 1 & 2 & & \\
\hline Universidade Estadual do Rio de Janeiro & & & & & 2 \\
\hline $\begin{array}{l}\text { Universidade Estadual Júlio de Mesquita Filho - } \\
\text { Bauru }\end{array}$ & 1 & & & 1 & \\
\hline $\begin{array}{l}\text { Universidade Estadual Júlio de Mesquita Filho - } \\
\text { Marília }\end{array}$ & & 1 & 1 & 2 & 1 \\
\hline $\begin{array}{l}\text { Universidade Estadual Júlio de Mesquita Filho - } \\
\text { Rio Preto }\end{array}$ & & & & & 1 \\
\hline Universidade de Santa Cruz do Sul & & & 1 & & \\
\hline Universidade Federal da Bahia & 1 & & 4 & & 3 \\
\hline Universidade Federal da Grande Dourados & & & & & 1 \\
\hline Universidade Federal da Paraíba & 1 & & & & 1 \\
\hline Universidade Federal da Paraíba/João Pessoa & 1 & 4 & 1 & 1 & \\
\hline Universidade Federal de Campina Grande & 1 & & & & \\
\hline Universidade Federal de Goiás & & & & 2 & 6 \\
\hline Universidade Federal de Mato Grosso & & & & 1 & 1 \\
\hline Universidade Federal de Minas Gerais & & 1 & 1 & 6 & \\
\hline Universidade Federal de Pelotas & & 1 & & & 2 \\
\hline Universidade Federal de Pernambuco & 3 & 3 & 1 & 2 & 2 \\
\hline Universidade Federal de Rondônia & & & & & 1 \\
\hline Universidade Federal de Santa Catarina & 4 & 6 & 4 & 11 & 7 \\
\hline
\end{tabular}

(continua) 
(conclusão)

\begin{tabular}{|c|c|c|c|c|c|}
\hline Universidade Federal de Santa Maria & 1 & 3 & 4 & & 3 \\
\hline Universidade Federal de São Carlos & 3 & 2 & 2 & 4 & 1 \\
\hline Universidade Federal de São Paulo & & & & & 1 \\
\hline Universidade Federal de Sergipe & & & & & 2 \\
\hline Universidade Federal de Uberlândia & & & 2 & 1 & \\
\hline Universidade Federal do Amazonas & & & 1 & 1 & 1 \\
\hline Universidade Federal do Ceará & & 1 & & 2 & \\
\hline Universidade Federal do Espírito Santo & 1 & 1 & 3 & 1 & \\
\hline Universidade Federal do Mato Grosso do Sul & & & & & 1 \\
\hline Universidade Federal do Pará & 1 & 2 & & & 1 \\
\hline Universidade Federal do Paraná & 2 & 2 & 1 & & 5 \\
\hline Universidade Federal do Piauí & & & 1 & 1 & \\
\hline Universidade Federal do Rio de Janeiro & 3 & 1 & 1 & 1 & 6 \\
\hline Universidade Federal do Rio Grande do Norte & & 1 & & & \\
\hline Universidade Federal do Rio Grande do Sul & 1 & 2 & 2 & 4 & 3 \\
\hline Universidade Federal Fluminense & & & & 1 & \\
\hline Universidade Grande Rio & & & & & 3 \\
\hline Universidade Luterana do Brasil & & 1 & 2 & & \\
\hline Universidade Metodista de Piracicaba & 1 & & & & 3 \\
\hline Universidade Metodista de São Paulo & & & & & 2 \\
\hline Universidade Presbiteriana Mackenzie & & 1 & & & \\
\hline $\begin{array}{l}\text { Universidade Regional do Noroeste do Estado do } \\
\text { Rio Grande do Sul }\end{array}$ & & & & & 1 \\
\hline Universidade São Francisco & 1 & & & & \\
\hline Universidade São Marcos & 1 & & & & \\
\hline Universidade Tuiuti do Paraná & & 1 & & & 2 \\
\hline Universidade Vale do Itajaí & & & & & 1 \\
\hline Universidade Veiga de Almeida & 1 & 2 & & & \\
\hline
\end{tabular}




\section{ANEXO 2}

TABELA 3 - ÁREAS DE CONCENTRAÇÃO POR ANO

\begin{tabular}{|c|c|c|c|c|c|}
\hline Área de concentração & 2007 & 2008 & 2009 & 2010 & 2011 \\
\hline Administração & & 1 & & & \\
\hline Antropologia Social & & & & 2 & \\
\hline Artes & & & & & 1 \\
\hline Bioquímica & & & & & 2 \\
\hline Ciência da Computação & 2 & 1 & 2 & 1 & 2 \\
\hline Ciência da Linguagem & & 1 & & & \\
\hline Ciência da Saúde & & & & & 4 \\
\hline Ciências da Informação & & 1 & & & 1 \\
\hline Computação & & 1 & & & \\
\hline Comunicação & 1 & & & & \\
\hline Crítica Cultural & & & & & 1 \\
\hline Desenho Industrial & & & 1 & & \\
\hline Design & & & & 1 & \\
\hline Design e Expressão Gráfica & & 2 & & & \\
\hline Distúrbio da Comunicação Humana & & & & & 1 \\
\hline Distúrbio do Desenvolvimento & & 1 & & & \\
\hline Educação & 9 & 21 & 33 & 24 & 42 \\
\hline Educação Ambiental & & & & & 1 \\
\hline Educação Escolar & & & 1 & 1 & \\
\hline Educação Especial & 5 & 3 & 4 & 2 & 5 \\
\hline Educação para Ciência e Ensino de Matemática & & 2 & & & 2 \\
\hline Educação: História, Política e Sociedade & & & & & 2 \\
\hline Engenharia e Gestão do Conhecimento & & & & & 1 \\
\hline Engenharia Elétrica & & 1 & & 1 & \\
\hline Ensino & & & & & 3 \\
\hline Ensino de Ciências da Saúde e do Ambiente & & 1 & & & \\
\hline Ensino de Ciências e Matemática & 2 & 3 & 4 & 3 & \\
\hline Ensino de Física & & & & & 1 \\
\hline Ensino de Tradução & & & & 3 & \\
\hline
\end{tabular}

(continua) 
(conclusão)

\begin{tabular}{|c|c|c|c|c|c|}
\hline Estudos da Linguagem & & & & & 1 \\
\hline Estudos Linguísticos e Literários em Inglês & & 1 & & 2 & \\
\hline Extensão Rural & & & & & 1 \\
\hline Fonoaudiologia & 3 & 4 & & 2 & 1 \\
\hline Geografia & & 1 & & & \\
\hline $\begin{array}{l}\text { Gestão do Conhecimento e Tecnologia da } \\
\text { Informação }\end{array}$ & & & & & 1 \\
\hline Informática & & 1 & & 1 & 1 \\
\hline Interdisciplinar & & & & & 3 \\
\hline Letras & 2 & 2 & 1 & 4 & 2 \\
\hline Letras, Cultura e Regionalidade & & & & & 1 \\
\hline Letras e Linguística & & & & 1 & 3 \\
\hline Língua Portuguesa & & & & & 1 \\
\hline Linguística & 2 & 8 & 7 & 9 & \\
\hline Linguística Aplicada & 1 & 6 & 4 & 2 & 3 \\
\hline Linguística, Letras e Artes & 2 & 1 & 4 & 1 & 5 \\
\hline Literatura & & & & 2 & \\
\hline Multidisciplinar & & & 1 & & 2 \\
\hline Odontologia & & & & 1 & \\
\hline Psicobiologia/Psicologia Fisiológica & 1 & & & & \\
\hline Psicolinguística & 1 & & & & \\
\hline Psicologia & 1 & 1 & & & 2 \\
\hline Psicologia Cognitiva & 1 & 1 & & & 1 \\
\hline Psicologia Escolar e do Desenvolvimento & & 1 & 1 & 1 & \\
\hline Psicologia Experimental & 2 & & 2 & & 1 \\
\hline Saúde Coletiva & 1 & & & 1 & \\
\hline Saúde Pública & & 1 & & 1 & \\
\hline Sociologia & & & 1 & & \\
\hline Teoria e Análise Linguística & 3 & & & 1 & \\
\hline Tópicos Específicos de Educação & 1 & & & & 1 \\
\hline
\end{tabular}




\section{ANEXO 3}

TABELA 4 - TEMÁTICAS ESCOLHIDAS E QUANTIDADE POR ANO

\begin{tabular}{|c|c|c|c|c|c|}
\hline Temática & 2007 & 2008 & 2009 & 2010 & 2011 \\
\hline A escrita da criança surda & & 2 & & & \\
\hline Alfabetização de surdos & & & & & 1 \\
\hline Ambiente virtual-Letras/Libras & & 1 & & & \\
\hline Ambientes virtuais de aprendizagem & & & 2 & 1 & \\
\hline Análise morfológica da Libras & & & 1 & & \\
\hline Animação virtual & & 1 & & & \\
\hline Aprendizagem da língua de sinais por ouvintes & & & 1 & & 1 \\
\hline Aquisição da leitura do português por surdos & & & & 1 & \\
\hline Aquisição da Libras & & & 1 & 1 & \\
\hline Aquisição da língua portuguesa escrita & & 4 & 2 & & \\
\hline Aquisição da linguagem por pessoas surdas & & & 1 & 1 & \\
\hline Aquisição do português por surdos & 3 & 4 & 3 & 1 & 6 \\
\hline Argumentação em Libras & & & 1 & 1 & \\
\hline Artes no ensino de surdos & & 1 & & & 2 \\
\hline Atendimento Educacional Especializado & 1 & & & & 1 \\
\hline Atendimento em saúde para surdos & & 1 & 1 & 1 & \\
\hline Atuação do tradutor intérprete & 1 & & 1 & 3 & 1 \\
\hline $\begin{array}{l}\text { Avaliação de alunos surdos com Altas } \\
\text { habilidades/superdotação }\end{array}$ & & & 1 & & \\
\hline Avaliação educacional & & 1 & & 1 & \\
\hline Bilinguismo & & 4 & & 2 & 4 \\
\hline Comparação entre português e LIBRAS & & & & & 1 \\
\hline Comunicação surdo - ouvinte & & & & 1 & \\
\hline Concepções de surdos sobre escolarização & & & & 2 & \\
\hline Concepções de surdos sobre o curso Letras LIBRAS & & & & & 1 \\
\hline Concepções ou representações sobre pessoas surdas & 1 & & 2 & 1 & 9 \\
\hline Construção de sentido por surdos & & & 1 & & \\
\hline Construção do conhecimento & & 1 & & & \\
\hline Currículo do curso de Fonoaudiologia & & & & 1 & \\
\hline Currículo na educação de surdos & & 1 & 2 & 1 & \\
\hline Desenvolvimento e surdez & & 1 & & 1 & \\
\hline
\end{tabular}

(continua) 
PAGNEZ, K. S.; GECIAUSKAS, C. S. O estado da arte de pesquisas sobre a educação...

(continuação)

\begin{tabular}{|c|c|c|c|c|c|}
\hline Diálogo entre mãe e filho/sinais caseiros & & 1 & & 1 & 1 \\
\hline Diversidade linguístico-cultural & & 1 & & & \\
\hline Educação Ambiental & 1 & & 1 & & 1 \\
\hline Educação Física e educação de surdos & 1 & 2 & & & \\
\hline Educação Superior/surdos & 2 & 1 & 1 & & 4 \\
\hline Empréstimo linguístico & & & & 1 & \\
\hline Ensino de Biologia & 1 & & & & \\
\hline Ensino de Ciências & & & 1 & 1 & \\
\hline Ensino de Física & 1 & & & & 1 \\
\hline Ensino de Inglês para alunos surdos & & & & 1 & \\
\hline Ensino de LIBRAS & 5 & & & 1 & 7 \\
\hline Ensino de Libras no Ensino Superior & & 1 & 1 & & \\
\hline Ensino de matemática & 2 & 1 & 3 & 3 & \\
\hline Ensino de música para surdos & & & 2 & & \\
\hline Ensino de química & & & & & 2 \\
\hline Ensino do português escrito a alunos surdos & 4 & 2 & 3 & 1 & 2 \\
\hline Ensino e aprendizagem de surdos & & 1 & 1 & 1 & \\
\hline Ergonomia da sala de aula & & & & 1 & \\
\hline Escolarização de pessoas surdas & & 2 & 4 & 1 & 23 \\
\hline Escrita da língua de sinais & & & 1 & & 1 \\
\hline Escrita do português por surdos & & & 1 & 5 & \\
\hline Estudos Culturais & & 1 & & 4 & 2 \\
\hline Estudos Surdos & 2 & & & 2 & 3 \\
\hline Formação de conceitos por alunos surdos & & & 2 & & \\
\hline Formação de professores para a diversidade & & & 1 & 2 & \\
\hline Formação do intérprete & & & 1 & 1 & \\
\hline Formação e atuação do intérprete & & & 1 & & \\
\hline Formação e educação de surdos & & 1 & & 3 & \\
\hline Funcionamento cognitivo & & & & & 1 \\
\hline Grupo de apoio à família de aluno surdo & & & 1 & & \\
\hline História da Educação de surdos & 2 & & 2 & & \\
\hline Humor surdo & & & & 1 & \\
\hline Identidade profissional do tradutor intérprete & & & & 1 & \\
\hline Identidade Surda & & & 1 & 1 & 2 \\
\hline Implante coclear & 1 & & & & \\
\hline Inclusão escolar & 1 & 5 & 2 & & \\
\hline Inclusão/exclusão social & & 1 & & & \\
\hline
\end{tabular}

(continua) 
(conclusão)

\begin{tabular}{|c|c|c|c|c|c|}
\hline Informática e ensino de surdos & 1 & 2 & 2 & 2 & \\
\hline Instrutor surdo & 2 & & & & \\
\hline Interação entre surdos e ouvintes & & 1 & & & \\
\hline Interação entre surdos e ouvintes em sala de aula & & & & 3 & \\
\hline Intérprete na escola & & & & & 1 \\
\hline Leitura do português por surdos & & & & & 1 \\
\hline Linguagem e subjetivação de surdos & & & 2 & & \\
\hline Linguística da LIBRAS & & 5 & 1 & 2 & 4 \\
\hline Literatura e deficiência & 1 & & & & \\
\hline Literatura Surda & & & & 1 & 2 \\
\hline Metalinguagem da Libras no ensino de português & & & 1 & & \\
\hline O brincar e a surdez & & 2 & & & \\
\hline Oposição ouvinte $\mathrm{x}$ surdo & & & & & 1 \\
\hline Pedagogia surda & & 1 & & & \\
\hline Perfil do aluno do curso Letras LIBRAS & & & & & 1 \\
\hline Políticas educacionais e surdez & 2 & 3 & 2 & & \\
\hline Prática docente no ensino de alunos surdos & & & & 3 & \\
\hline Proficiência da Libras & & & & 1 & \\
\hline Psicologia e surdez & 4 & 2 & & & \\
\hline Recursos didáticos para o ensino de LIBRAS & & & 1 & 2 & 1 \\
\hline Recursos metodológicos no ensino de surdos & 1 & 1 & & & 1 \\
\hline Representação cultural do professor surdo & & & 1 & & \\
\hline Sexualidade & & & 1 & & 1 \\
\hline Sociolinguística & & 2 & & & \\
\hline Tecnologia Assistiva e surdez & & & 2 & & 1 \\
\hline Tecnologias da Informação e LIBRAS & & & & 1 & 1 \\
\hline Tradução & & 1 & & 2 & \\
\hline Trajetória de surdos & 1 & 1 & & & 3 \\
\hline Uso da língua inglesa por surdos & & 1 & 1 & 1 & \\
\hline Uso de prótese auditiva & 1 & & & & \\
\hline Validação de instrumento em LIBRAS & & & & 1 & 3 \\
\hline Variação Linguística & & & & & 1 \\
\hline Visualidade e educação de surdos & & 1 & 1 & 1 & 2 \\
\hline
\end{tabular}




\section{ANEXO 4}

TABELA 5 - TIPOS DE PESQUISA POR ANO E QUANTIDADE

\begin{tabular}{|c|c|c|c|c|c|}
\hline Tipologia & 2007 & 2008 & 2009 & 2010 & 2011 \\
\hline Análise Descritiva & & 1 & & & 3 \\
\hline Análise Lexical & & & & & 1 \\
\hline Análise Temática & & & & & 1 \\
\hline Descritivo analítico & & & 1 & & \\
\hline Documental & & & 1 & & \\
\hline Estudo de caso & & 4 & 4 & 4 & 2 \\
\hline Etnográfica & 2 & 5 & 1 & 5 & 4 \\
\hline Experimental & 1 & & & & 6 \\
\hline Exploratória & & 2 & & 1 & \\
\hline Exploratório-descritiva & 1 & 1 & & & \\
\hline Heurística & & 1 & & & \\
\hline Histórico-documental & & 1 & & & 1 \\
\hline Intervenção crítica & & & 1 & & \\
\hline Investigação-ação & & & 2 & & \\
\hline Investigação narrativa & & & 1 & & \\
\hline Levantamento & 2 & & & & \\
\hline Longitudinal & 1 & & & & \\
\hline Modelo Microgenético & & & & & 1 \\
\hline Participante & & 4 & & & \\
\hline Pesquisa-Ação & 1 & 1 & 2 & & 2 \\
\hline Pesquisa Bibliográfica & & & 1 & & 1 \\
\hline Pesquisa Empírica & 1 & & & & 1 \\
\hline Qualitativa & 3 & 7 & 8 & 6 & 9 \\
\hline Qualitativa e quantitativa & 1 & 2 & & 1 & \\
\hline Revisão Bibliográfica & & & & & 1 \\
\hline Sem descrição & 31 & 34 & 47 & 54 & 69 \\
\hline
\end{tabular}




\section{ANEXO 5}

TABELA 6 - REFERENCIAIS TEÓRICOS RELACIONADOS À SURDEZ E QUANTIDADE DE CITAÇÕES POR ANO

\begin{tabular}{|c|c|c|c|c|c|}
\hline Referencial & 2007 & 2008 & 2009 & 2010 & 2011 \\
\hline Bakhtin & & & 1 & 1 & 4 \\
\hline Capovilla,César & 2 & & 2 & 1 & \\
\hline Capovilla, Alessandra & 2 & & 2 & 1 & \\
\hline Estudos Culturais & 1 & & & 1 & 3 \\
\hline Estudos Surdos & & 1 & & & 3 \\
\hline Felipe & & 1 & & & 1 \\
\hline Fernandes & & & & 1 & \\
\hline Góes & & 1 & & 1 & \\
\hline Goffman & & 1 & & & \\
\hline Johnson & 2 & & & & \\
\hline Karnopp & & 1 & 1 & & 1 \\
\hline Klein & & & & & 1 \\
\hline Lacerda & & & & 2 & 1 \\
\hline Larossa & & 1 & & & \\
\hline Lodi & & & 1 & & \\
\hline Lunardi & & 1 & & & 1 \\
\hline Luria & & 2 & & 1 & \\
\hline Perlin & & 1 & 2 & 1 & 2 \\
\hline Quadros & 1 & 2 & 1 & 2 & 5 \\
\hline Raphael & & 1 & 2 & & 1 \\
\hline Sá & & & 1 & 1 & \\
\hline Sacks & & & 1 & 1 & \\
\hline Salles & & 1 & & & \\
\hline Silveira & & & & & 1 \\
\hline
\end{tabular}

(continua) 
PAGNEZ, K. S.; GECIAUSKAS, C. S. O estado da arte de pesquisas sobre a educação...

(conclusão)

\begin{tabular}{|c|c|c|c|c|c|}
\hline Skliar & & 3 & 3 & 5 & 4 \\
\hline Soares & & & 1 & 1 & 1 \\
\hline Souza & & 1 & 1 & 1 & \\
\hline Strobel & & & 1 & 1 & 2 \\
\hline Suppalla & & & & & 1 \\
\hline Sutton & & & & & 2 \\
\hline Stumpf & & & 1 & & \\
\hline Thoma & 2 & 8 & 6 & 7 & 10 \\
\hline Vygotsky & 36 & 44 & 54 & 56 & 54 \\
\hline $\begin{array}{c}\text { Sem referências da área de } \\
\text { surdez }\end{array}$ & & & & & 1 \\
\hline
\end{tabular}

